\title{
Tinnitus characteristics at high-and low-risk occupations from occupational noise exposure standpoint
}

Mehdi Asghari

\begin{abstract}
Introduction: The aim of the present study was to compare tinnitus characteristics in high- and low-risk occupations from the occupational noise exposure standpoint, considering demographic data, hearing loss and concomitant diseases.

Methods: Demographic data, characteristics of tinnitus, hearing and concomitant diseases were recorded in the questionnaires. Their pure tone air conduction thresholds were determined using a double-channel diagnostic Audiometer and the Bone Conduction was assessed using a B-71 bone vibrator.

Results: Totally, $6.3 \%$ subjects (6.8\% high-risk group and $5.6 \%$ low-risk group) had subjective tinnitus, mainly as whistling sound. In the high-risk group, tinnitus was mainly left-sided (41.18\%) and hearing loss was mild. Bilateral tinnitus (52.63\%) and slight hearing loss were observed predominantly in the low-risk group.
\end{abstract}

Conclusions: The study showed higher incidence of tinnitus in high-risk professions regarding with occupational noise exposure.

Keywords: Tinnitus; Loudness; Hearing loss; Noise exposure; High-risk occupations. 


\section{INTRODUCTION}

Tinnitus is a sound sensation in the ears or head in the absence of an external auditory or electrical source. It has been recognized as a major public health issue for people is occupationally exposed to hazardous noise 1,2 . In recent decades, rapid industrialization has led to a remarkable interest in harmful effects of occupational noise exposure in the some workplaces ${ }^{3,4}$. Literature indicates the high level of noise exposure as a health risk factor of workers may interfere with their performance through causing high blood pressure, anxiety and especially hearing loss and tinnitus ${ }^{5}$. Current knowledge about the pathophysiology of tinnitus is still very limited, but there have always been theories about it. Tinnitus can be caused by progressive destruction of the cochlea and changes in the rate at which the auditory nerve fibre fires due to the energy generated by the high sound pressure. Tinnitus might interfere with speech communication, intelligibility and localization of sound sources at workplaces. It has been considered as a workplace safety threat, even in individuals with normal hearing ${ }^{6}$. However, tinnitus itself is not a disease but can be a symptom of other disorders, including auditory, psychogenic, neurologic, cardiovascular, dental, metabolic diseases or even ototoxic drugs and other unknown causes ${ }^{7}$. Tinnitus is generally triggered by hearing loss and very often by exposure to intense non-pulsating and continuous noise and affecting by Noise-Induced Hearing Loss (NIHL) ${ }^{8-10}$. It is auditory phenomenon associated with professions exposed to high noise levels ( $\geq 85 \mathrm{~dB} A$ ) or long exposure times to noise such as operating engineers, construction, metal mining, manufacturing and agricultural industry 11 . Some studies revealed that $18 \%$ of the population in industrialized countries are mildly affected by tinnitus 8,11. Also, the tinnitus prevalence has been reported $29.7 \%, 23.3 \%, 11.9 \%$, and $10 \%$ among noise-exposed workers in the United Kingdom, Singapore, Japan and $\mathrm{KSA}$, respectively,12-15. In addition to high-level noise exposure was suggested as a primary cause of hearing loss; the characteristics of tinnitus, demographic and health factors may also be as another contributing factors 16. Since, there is no report specifically for tinnitus in the OSHA program due to lack of objective diagnosis criteria. So, when tinnitus is an only complaint, there is no supportive care for tinnitus treatment or rehabilitation program by organizations and health Insurance companies ${ }^{17}$. Because the relationship between tinnitus and noise exposure and other contributors in incidence of tinnitus like demographic characteristics, hearing loss and concomitant diseases is not well understood and not considered in the previous studies, the present study aimed to compare the incidence of tinnitus in occupations with high risk and low risk from the perspective of occupational noise exposure while considering demographic features, hearing loss and other concomitant diseases.

\section{MATERIALS AND METHODS}

This is a cross-sectional study with a descriptive-analytical approach, which was performed on 836 participants aged
20 to 60 years referred to XXX Occupational Medicine Centers in 2018, Arak, Iran. Inclusion criteria included age $\geq 18$, at least a fifth grade education, wok experience $\geq 1$-year full time, and no conductive component and tympanic membrane inflammation in the Otoscopy. Subjects with a history of psychiatric or psychological disorders, long-treatment with ototoxic drugs, and family history of hearing loss, acoustic trauma, and pulsating tinnitus were excluded. After obtaining informed consent, a study baseline questionnaire was completed, including questions pertaining to demographic characteristics, occupation type, work experience, hearing loss and tinnitus complaints. The presence of tinnitus was evaluated by a yes-no question. Also, comorbidities such as diabetes, hypertension, cardiovascular, metabolic and dental diseases was investigated. Then, all participants received a free otoscopic and physical examination by professional physician. Data were recorded in a questionnaire designed for this study. After that, all the information about tinnitus (quality, laterality and duration) was analyzed. All the other stages were performed by a trained audiologist. In the present study, tinnitus was defined as the sensation of sound perceived in the head or ears without stimulus lasting for at least 3 months. Subjects were divided into two groups based on risk of their jobs according to noise exposure ${ }^{5}$. The first group consisted of those involved in one of the highrisk occupations in terms of NIHL ( $\geq 85 \mathrm{~dB} A)(n=497)$, including military jobs ${ }^{18}$, carpenters ${ }^{19}$, manufacturing workers ${ }^{20}$, driver, rail roaders ${ }^{21}$, construction workers ${ }^{22}$ and aluminum work industry ${ }^{19}$. The lower-risk group (with office-like noises e.g. Telephone or chatting or usual background noise) consisted of entrepreneurs, company managers or executives, hospital staff and administrators, and healthcare professionals ${ }^{4,6}$. Pure tone Air Conduction thresholds were determined in each ear at 0.25, 0.5, 1, $2,3,4,6$, and $8 \mathrm{khz}$ using a double-channel diagnostic Audiometer (TDH-39 Supra-aural headphone) and the Bone Conduction was assessed at 0.25-4 khz using a B-71 bone vibrator. The evaluations were performed at least 14 hours after the acoustic rest. Hearing threshold was defined as abnormal if pure-tone threshold average at $0.5,1$, 2,3 and $4 \mathrm{khz}$ was $\geq 25 \mathrm{~dB}$ HL in the worse ear3,4.Data were analyzed by SPSS software version 21 . Chi-square test was used to analyze differences of categorized demographic variables and tinnitus characteristics between the high - and low -risk groups, and Independent Samples t-test and oneway ANOVA were used to determine statistical differences for two and more quantitative variables, respectively. A $p$-value less than 0.05 (typically $\leq 0.05$ ) was considered statistically significant.

\section{RESULTS}

\section{Characteristics of study population}

836 subjects aged 20-60 years (769 males (92\%) and 67 females (8\%)) with work experience of $1-30$ years $(10.76$ \pm 7.4.) Were studied. The demographic characteristics are presented in Table1. Of the $\mathbf{8 3 6}$ patients that were 
included in this study, $339(40.55 \%)$ had no history of excessive noise exposure, and 497 (59.45\%) had history of exposure to intense occupational noise. The mean age was $36.6 \pm 8.85$ in the high-risk and $36.39 \pm 9.8282$ low-risk groups. No significant statistical difference was reported in age between groups with independent $T$ test $(p=0.63)$. The total number of males was significantly greater in the studied population (>90\%) $(97 \%$ in highrisk group VS. $85 \%$ in low-risk group). According to Table 1 , most of subjects were in their 20 to 40 years $(\sim 75 \%$ for both groups) and were Diploma and under diploma ( $\sim 80 \%$ for high-risk group). There was no significant difference between the two groups in terms of education level $(P=0.07)$. The mean work experience was lower in the high-risk group; with no statistically significant difference between two groups $(p=0.16)$. The majority of participants lived in urban areas (97\%) $(98.6 \%$ in high-risk group vs. $94.7 \%$ in low-risk group).

\section{Tinnitus and hearing loss features}

The overall prevalence of tinnitus was $6.3 \%$ (5.6\% in lowrisk group and $6.84 \%$ in high-risk group). As expected, the prevalence of tinnitus was higher in high-risk group than low-risk group. All patients had subjective tinnitus. $50.95 \%$ of participants was described their tinnitus as a whistle sound. There is significant statistical association between occupation type and the occurrence of tinnitus $(P<0.0001)$. All affected subjects had subjective tinnitus with or without hearing loss. Hearing loss prevalence was reported $34.69 \%$ (35.31\% in low-risk group and $33.92 \%$ in high-risk group); with no significant difference between two groups $(p=0.16)$. Prevalence of hearing loss, and tinnitus among occupation groups is presented in Table 2. Among the occupants, the highest risk of tinnitus occurrence was observed in hospital staff (PR $=0.13$ ) in the low-risk group and in railway personnel and mechanics $(P R=0.11)$ in the high-risk group. Other services (e.g. Security and other services) were more likely to report hearing loss in the low-risk group $(P R=0.63)$ and professionals $(P R=0.48)$ respectively, compared with drivers $(P R=0.5)$ and military personnel $(P R=0.4)$ in the high-risk group as shown in Table 2. One-way ANOVA indicated no significant statistical difference with regard to tinnitus occurrence, tinnitus location, hearing loss incidence and hearing loss degree among occupation groups in both low-risk and high-risk groups ( $p>0.05)$.

\section{Tinnitus and demographic characteristics}

53 subjects with tinnitus included 19 subjects (12 males, 7 females) in the low risk group and 34 subjects (30 males, 4 females) in the high-risk group. The descriptive statistics related to tinnitus are shown in Table 3 . There was significant difference between groups in occurrence of tinnitus using chi-square $(p=0.001)$.A higher proportion of subjects experienced tinnitus with hearing loss in the high-risk group than low-risk group (32.3\% vs. $24.2 \%)$. Average duration of tinnitus was lower in the low-risk group than in the high-risk group $(3.01 \pm 2.90$ vs.5.11 \pm 4.67), with significant difference between groups $(p=0.002)$. Tinnitus was mostly unilateral and left-sided in $41.85 \%$ of subjects in the high-risk group and bilateral in $52.63 \%$ of cases in the low-risk group; difference was not statistically significant between groups $(p=0.56)$. Subjects with tinnitus had greater degree of hearing loss in the high-risk group than low-risk group; with no significant difference in hearing loss $(p=0.51)$. In the low risk group, tinnitus often was accompanied by slight hearing loss in comparison with mild hearing loss in the high-risk group. In other words, the mean hearing threshold of intense noise-exposed subjects was worse than other group; no statistically significant difference was reported between groups $(p=0.27)$. No significant relationship was found between tinnitus and hearing loss using the chi-square test $(p=0.34)$. In this study, the highest prevalence of audiogram shape was related to high frequency loss $(47.6 \%)$ in the right ear in both groups. (High-risk (15.1\%) vs. Low-risk $(20.1 \%)$ ); with no significant difference between groups $(p=0.12)$.

\section{Tinnitus and additional comorbidity}

$229(27.39 \%)$ of all subjects had at least one comorbid diseases $(27.72 \%$ in low-risk groups and $27.16 \%$ in highrisk group). The frequencies of concomitant diseases in our population are shown in Figure 1. The results showed

Table 1: The distribution of demographic characteristics among 836 subjects in high- and low- risk groups.

\begin{tabular}{|c|c|c|c|c|c|c|}
\hline \multicolumn{2}{|c|}{ Demographic characteristics } & \multicolumn{2}{|c|}{$\begin{array}{l}\text { Low-risk Group } \\
\quad(n=339)\end{array}$} & \multicolumn{2}{|c|}{$\begin{array}{l}\text { High-risk Group } \\
\quad(n=497)\end{array}$} & \multirow[t]{2}{*}{ P-value } \\
\hline & & $\mathrm{N}$ & Percent & $\mathrm{N}$ & Percent & \\
\hline \multirow{2}{*}{ Age } & $\leq 40$ years $(n=620)$ & 256 & $75.52 \%$ & 372 & $74.8 \%$ & \multirow{2}{*}{ NS } \\
\hline & $40-60$ years $(n=199)$ & 83 & $24.48 \%$ & 125 & $25.2 \%$ & \\
\hline \multirow{4}{*}{ Sex } & Female & 52 & $15.33 \%$ & 15 & $3 \%$ & \multirow{2}{*}{ NS } \\
\hline & Male & 287 & $84.67 \%$ & 482 & $97 \%$ & \\
\hline & Under diploma & 92 & $27.14 \%$ & 227 & $45.67 \%$ & \multirow{6}{*}{ NS } \\
\hline & Diploma & 69 & $20.36 \%$ & 176 & $35.41 \%$ & \\
\hline Education & Associate Degree & 29 & $8.55 \%$ & 36 & $7.24 \%$ & \\
\hline \multirow[t]{3}{*}{ level } & Bachelor's Degree & 89 & $26.25 \%$ & 50 & $10.06 \%$ & \\
\hline & Master's Degree & 31 & $9.15 \%$ & 6 & $1.21 \%$ & \\
\hline & $\mathrm{PhD}$ & 29 & $8.55 \%$ & 2 & $0.41 \%$ & \\
\hline $\begin{array}{l}\text { Work experience in years } \\
\text { (Mean (SD)) }\end{array}$ & & \multicolumn{2}{|c|}{$19.10(10.10)$} & \multicolumn{2}{|c|}{$17.67(8.75)$} & NS \\
\hline
\end{tabular}


Table 2: Prevalence of hearing loss, and tinnitus among occupation groups.

\begin{tabular}{|c|c|c|c|c|c|c|}
\hline Groups & Job Title & No. & $\underset{\mathbf{N}}{\text { Tinnitus }}$ & $\begin{array}{c}\text { Gender } \\
\text { (male) }\end{array}$ & Mean Age (year) & $\begin{array}{c}\text { Hearing loss } \\
\mathbf{N}\end{array}$ \\
\hline \multirow{8}{*}{$\begin{array}{l}\text { Low-risk group } \\
\qquad N=339\end{array}$} & Office staff & $154(45.43 \%)$ & $12(7.79 \%)$ & 124 & 35.74 & $46(29.87 \%)$ \\
\hline & Hospital staff & $23(6.78 \%)$ & $3(13.04 \%)$ & 12 & 33.82 & $6(26.1 \%)$ \\
\hline & Manager & $23(6.78 \%)$ & $2(8.69 \%)$ & 20 & 37.56 & $6(26.1 \%)$ \\
\hline & Professionals & $31(9.15 \%)$ & $1(3.22 \%)$ & 28 & 36.93 & $15(48.38 \%)$ \\
\hline & Machine operator & $27(7.96 \%)$ & $1(3.70 \%)$ & 25 & 36.55 & $8(29.63 \%)$ \\
\hline & Lab quality control & $24(7.80 \%)$ & $0(0 \%)$ & 21 & 34.08 & $10(41.66 \%)$ \\
\hline & Electrician & $38(11.22 \%)$ & $0(0 \%)$ & 38 & 35.63 & $12(31.58 \%)$ \\
\hline & Other Services & $19(5.60 \%)$ & $0(0 \%)$ & 19 & 36.84 & $(63.15 \%) 12$ \\
\hline \multirow{10}{*}{$\begin{array}{l}\text { High-risk group } \\
\qquad N=497\end{array}$} & P-value* & & NS & NS & NS & NS \\
\hline & Worker & $152(30.58 \%)$ & $16(10.52 \%)$ & 143 & 37.87 & $55(36.18 \%)$ \\
\hline & Military personnel & $75(15.1 \%)$ & $3(4 \%)$ & 72 & 36.67 & $30(40 \%)$ \\
\hline & Aluminum worker & $64(12.88 \%)$ & $2(3.12 \%)$ & 31 & 35.33 & $23(35.94 \%)$ \\
\hline & Railway personnel & $17(3.42 \%)$ & $2(11.76 \%)$ & 17 & 31.06 & $5(29.41 \%)$ \\
\hline & Blacksmith & $95(19.11 \%)$ & $7(7.37 \%)$ & 95 & 36.85 & $26(27.37 \%)$ \\
\hline & Mechanic & $34(6.84 \%)$ & $4(11.76 \%)$ & 34 & 36.59 & $13(38.23 \%)$ \\
\hline & Driver & $24(4.83 \%)$ & $0(0 \%)$ & 24 & 40.41 & $12(50 \%)$ \\
\hline & Assembler & $36(7.24 \%)$ & $0(0 \%)$ & 33 & 32.80 & $11(30.55 \%)$ \\
\hline & P-value & & NS & NS & NS & NS \\
\hline
\end{tabular}

*Pearson's $x^{2}$ test was done for categorical variables and one-way analysis of variance for continuous variables; NS: Not significant.

Table 3: Distribution of tinnitus characteristics in high- and low-risk groups.

\begin{tabular}{|c|c|c|c|c|}
\hline \multicolumn{2}{|c|}{ Tinnitus features } & \multirow{2}{*}{$\begin{array}{l}\text { Low-risk group } \\
\qquad \begin{array}{c}\mathbf{N}=19 \\
2(10.53 \%)\end{array}\end{array}$} & \multirow{2}{*}{$\begin{array}{l}\text { High-risk group } \\
\qquad \begin{array}{c}\mathbf{N}=\mathbf{3 4} \\
8(23.52 \%)\end{array}\end{array}$} & \multirow[t]{2}{*}{ P-value } \\
\hline & Right-sided & & & \\
\hline \multirow[t]{2}{*}{ Laterality } & Left-sided & $7(36.84 \%)$ & $14(41.18 \%)$ & \multirow[t]{2}{*}{ NS } \\
\hline & Bilateral & $10(52.63 \%)$ & $12(35.30 \%)$ & \\
\hline \multirow{3}{*}{ Age (year) } & $\leq 40$ & $6(31.57 \%)$ & $14(41.17 \%)$ & \multirow{3}{*}{ NS } \\
\hline & $40-60$ & $13(68.43 \%)$ & $20(58.83 \%)$ & \\
\hline & $<5$ & $10(52.63 \%)$ & $29(85.29 \%)$ & \\
\hline \multirow[t]{2}{*}{ Tinnitus duration (year) } & $5-10$ & $3(15.79 \%)$ & $3(8.83 \%)$ & \multirow[t]{2}{*}{$\mathrm{P}<0.001$} \\
\hline & $>10$ & $6(31.58 \%)$ & $2(5.88 \%)$ & \\
\hline Hearing loss & & $15(78.9 \%)$ & $28(82.4 \%)$ & NS \\
\hline
\end{tabular}

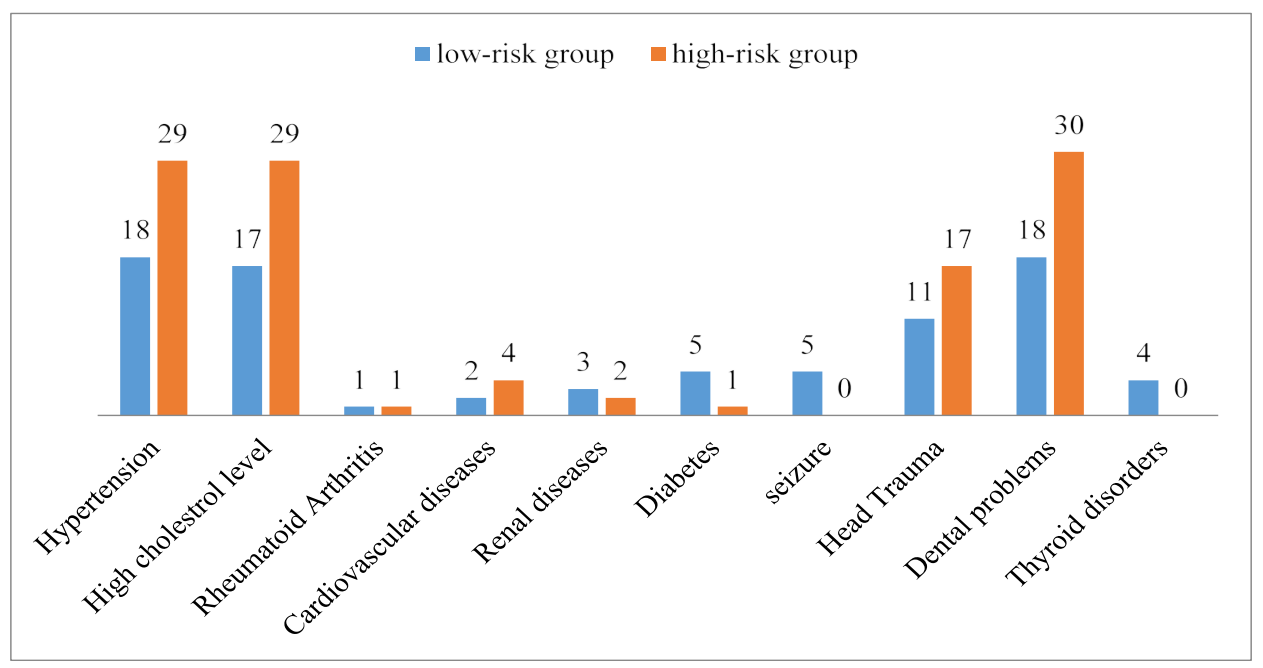

Figure 1: The frequency of concomitant diseases in high- and low-risk groups.

that dental problems, hypertension and high cholesterol level were the most concomitant medical problems in both groups with higher incidence in the high-risk group (Fig.1). All subjects with a history of rheumatoid arthritis, thyroid disorders, head trauma, epilepsy and diabetes -despite the small subject number s-affected by tinnitus in this study, there was no significant difference in comorbidity between the two groups except for kidney disease $(p>0.05)$. Significant difference was observed between groups in hypertension, cardiovascular diseases, 
diabetes, head trauma and dental problems. Multiple logistic regression analysis was also performed from the data of 836 subjects. Tinnitus was the dependent variable (y) and noise exposure, age, gender, hypertension, and diabetes were the independent variables $(x)$. None of the studied variables has no significant effect on prevalence of tinnitus (Exposure to noise, $p=0.058$; age, $p=$ 0.856 ; gender, $p=0.754$; hypertension, $p=0.300$; and diabetes, $p=0.442$ ).

\section{DISCUSSION}

This study is the first study to comprehensively and comparatively investigate the prevalence of tinnitus amongst different occupations in Iran. A relationship between tinnitus, sensorineural hearing loss, and occupational noise has been emphasized in previous studies. However, different aspects of this relationship have not yet been identified and remained controversial 5. We found higher prevalence of tinnitus among subjects exposed to high level of noise (6.84\%) than in low-risk group $(5.6 \%)$ which was in line with tinnitus prevalence rates between $4.4 \%$ and $20.1 \%$ reported in previous studies ${ }^{23}$. It was lower than in other previous studies reported $12-70.4 \%$ prevalence rate in the high-risk group and similar to $2 \%-14.4 \%$ in the low-risk group ${ }^{24,25}$. Because of differences in age groups and tinnitus definition, it is difficult to compare prevalence between studies. Data showed that tinnitus occurrence differed among occupations. Subjects exposed to excessive occupational noise 1.78 times were more likely to experience tinnitus compared with non-exposed ones. Significant association was observed between occupations and occurrence of tinnitus which is consistent with results of logistic regression models in Fredriksson's study ${ }^{26}$. In fact, negative effect of prolonged exposure to noise on outer hair cells and auditory nerve can lead to tinnitus. Majority of cases had unilateral left-sided tinnitus in the high-risk group. These findings are contrary to bilateral tinnitus has been reported in the high-risk group ${ }^{27}$. Handedness and external factors such as occupation, type of facility and process, the location of the machinery or tools have been suggested as risk factors of developing tinnitus. In the low-risk group, tinnitus was more perceived in the head or both ears, which was consistent with other studies ${ }^{28}$. There was no significant difference between the two groups in terms of the tinnitus location. Subjects in the high-risk occupations experienced significant longer duration of tinnitus and the difference was statistically significant between two groups. Tinnitus was found in both sexes in this study with higher male prevalence of tinnitus, more frequently in the high-risk group. This results support previous research results by Otoghile et al. showing higher proportion of males are more likely to be exposed to hazardous occupational noise and have a higher prevalence of hearing loss than female workers which is likely due to the traditional employment of men in noisy industries such as construction, mining, lumber, and manufacturing2. In this study, females mainly employed in the low-risk occupations, such as administration and hospital activities. Tinnitus occurs most often in the middecades of life, confirming the results of Martines et al. Also, it has been mentioned that tinnitus incidence usually remains unchanged and reaches plateau in the 60 s or 70 s $^{29}$. No association was found between education level and tinnitus, which is confirmed by Nondahl's findings while others have reported an inverse relationship26. In the present study, high-risk group had a higher prevalence of hearing loss than the low-risk group (35.31\% vs. $33.92 \%$ ) which was almost similar to that in Switzerland (31\%) but it was significantly higher than rates in Norway (16.1\%), United States (16.1\%) and South Korea (10\%) (23). It indicates that this trend may be accelerated by noisy environments. In the subgroups, hearing loss was more common in drivers and military personnel in the high-risk group which is in accordance with Moalemi and Haji Abolhassan study ${ }^{21}$. Also, Wells et al. found that participation in wars increase the risk of experiencing hearing loss to $63 \% 18$. $83.3 \%$ of tinnitus cases had hearing loss on conventional Audiometry which was higher than that previously reported and confirm the results of palmer et al. ${ }^{3,5}$. No significant difference was reported in hearing loss occurrence and degree between groups. Hearing loss was found most at higher frequencies. Other researchers have reported similar findings ${ }^{14,19}$; In contrast, there was no association between tinnitus and audiogram slope contrary to the results of Konig's et al. Reported increased occurrence of tinnitus with increasing audiogram slope ${ }^{24}$. Correlation was found between age, tinnitus duration, and poorer hearing thresholds. This is in line with findings reported by Tikka et al. ${ }^{15,29}$. An unexpected and very interesting finding in this study was the relatively high prevalence of tinnitus among hospital staff and management-related occupations. The prevalence of hearing loss was high in other services e.g. Securities and professionals which were considered as low- risk groups in terms of experiencing noise-induced auditory problems19. It seems that despite evidence linking tinnitus and noise, few studies have been conducted to determine exact prevalence of tinnitus in these specific occupational groups. Long exposure noise in non-industrial or officebased workplaces, wireless phone over use, sedentary jobs, hospital noisy equipment, and alarms and even can be considered as a trigger of tinnitus and hearing loss. So, this adds to the appeal of further research to determine and clarify the distinct role of risk factors. Another noteworthy issue is the emphasizing on the importance of noise level measurement; raising the awareness level of the occupants about using hearing protection devices and conservative programs that has attracted much attention for provoking anti-noise and preventive behaviors in higher risk professions but are not considered vital enough for non-industrial or small-scale settings ${ }^{30}$. Cardio-vascular disease, hypertension, diabetes mellitus and high cholesterol levels have been proposed as non-auditory consequences of noise and risk factors of tinnitus as well 5,19. However, the associations of these factors with tinnitus are controversial. In our sample, we 
found no significant difference in concomitant diseases between the two groups, except for renal diseases. So, such analysis should be interpreted with caution due to the small number of cases.

Study limitations: Investigating tinnitus is too complicated, expensive and time consuming among different occupational groups. The first limitation of the study was the lack of information on the exact noise level in the workplaces due to low budgetary constraints and variety of professions. So, subjects were selected for the groups based on the demographic findings noted in previous large studies and the recommendation of the National Institute of Occupational Safety and Health (NIOSH). Second, psychoacoustic evaluation was not performed for tinnitus loudness and pitch. Third, hearing test and tinnitus exam was limited to conventional behavioral pure-tone audiometry and did not assess the Outer hair cells function using OAE or high frequency audiometry. Fourth, the impact of external factors such as noise of vehicles, traffic, and recreational activities was not considered due to practical constraints. However, the literature on the relationship between environmental noise exposure and hearing loss / tinnitus is very limited.

\section{CONCLUSION}

We found that individuals in the high-risk group were predominantly males and experienced longer left-sided tinnitus and poorer hearing thresholds. There was a significant relationship between occupation groups and tinnitus. There was no association between demographic factors and tinnitus. Further clinical research is necessary to investigating the cumulative effects of occupational hazards and relevant risk factor due to the aforementioned difficulty in defining the causes of tinnitus and the numerous comorbidities present in this population. Novel findings have been reported on hearing-related symptoms in relation to noise exposure in low-risk and high-risk groups. The impact of external factors in subsequent studies should be considered due to the higher prevalence of tinnitus in some low-risk occupations. So, these findings emphasize on repeating and redesigning of more targeted studies on the auditory system among different occupations with regard to advances in technology including new machines and settings. The knowledge gained from this study provides a foundation for developing workplace health policy changes for some low-risk occupations.

\section{CONFLICT OF INTEREST}

The authors declares no conflict of interest

\section{ETHICS APPROVAL}

Ethical approval for this study was obtained from Arak University of Medical Sciences (330.1395IR.ARAKMU.REC).

\section{ACKNOWLEDGEMENT}

We thank the esteemed Vice Chancellor for Research of Arak University of Medical Sciences (Grant. No: 2971) who helped us in carrying out this project and we sincerely thank Mr. Rahim Gorji for his valuable cooperation.

\section{REFERENCES}

1. Hong O, Chin DL, Phelps S, Joo Y. Double Jeopardy: Hearing loss and tinnitus among noise-exposed workers. Workplace Health Saf. 2016;64:235-42.

2. Otoghile B, Idugboe OJ, Totyen EL, Otoghile CC. Tinnitus: Its pattern among noise exposed laborers. Int $\mathrm{J}$ Med Health Res. 2018;4:44-6.

3. Gilles A. Noise-induced Tinnitus in Adolescents: Prevalence, Detection \& Prevention: Proefschrift. Universiteit Antwerpen, Faculteit Geneeskunde en Gezondheidswetenschappen. 2014;1:1-6.

4. Engdahl B, Tambs K. Occupation and the risk of hearing impairment results from the Nord-Trøndelag study on hearing loss. Scand J Work Env Hea. 2010;36:250-7.

5. Ralli M, Balla MP, Greco A, Altissimi G, Ricci P, Turchetta R, et al. Work-related noise exposure in a cohort of patients with chronic tinnitus: Analysis of demographic and audiological characteristics. Int J Environ Res Pub Health. 2017;14:1035.

6. Cantley LF, Galusha D, Cullen MR, Dixon-Ernst C, TessierSherman B, Slade MD, et al. Does tinnitus, hearing asymmetry, or hearing loss predispose to occupational injury risk? Int J Audiol. 2014;54:30-6.

7. Kroon H. Collaborative audiological and psychological intervention where tinnitus and hearing loss co-exist (Doctoral dissertation, University of Pretoria). 2017.

8. Engdahl B, Krog NH, Kvestad E, Hoffman HJ, Tambs K. Occupation and the risk of bothersome tinnitus: results from a prospective cohort study (HUNT). BMJ Open. 2012;2:e00051-62.

9. Mazurek B, Olze H, Haupt $H$, Szczepek AJ. The More the Worse: the Grade of Noise-Induced Hearing Loss Associates with the Severity of Tinnitus. Int $\mathrm{J}$ Env Res Pub Health. 2010;7:3071-9.

10. Nageris BI, Attias J, Raveh E. Test-retest tinnitus characteristics in patients with noise-induced hearing loss. Am J Otolaryng. 2010;31:181-4.

11. Otoghile B, Otoghile CC, Ariyibi NO. Effects of Tinnitus on Quality of Life Among Sawmill Workers. Int J Otorhinolaryngol 2018;4:31.

12. Le TN, Straatman LV, Lea J, B W. Current insights in noiseinduced hearing loss: a literature review of the underlying mechanism, pathophysiology, asymmetry, and management options. J Otolaryngol-Head N. 2017;46:1-6.

13. Saleem AH, Alkharboush G, Almazyed O, AlHilal S, Alnajashi I, Al-Andejani T, et al. Prevalence of Hearing Loss and Tinnitus with Correlation to the Usage of Protective Hearing Equipment among Airport Workers. Journal of Nature and Science of Medicine. 2018;1:31-6.

14. Soalheiro M, Rocha L, do Vale DF, Fontes V, Valente D, Teixeira LR. Speech recognition index of workers with tinnitus exposed to environmental or occupational noise: a comparative study. J Occup Med Toxicol. 2012;7:26. 
15. Tikka C, Verbeek JH, Kateman E, Morata TC, Dreschler WA, Ferrite S. Interventions to prevent occupational noiseinduced hearing loss. Cochrane Database Syst Rev. 2017;7:63-96.

16. Sheppard A, Hayes SH, Chen GD, Ralli M, Salvi R. Review of salicylate-induced hearing loss, neurotoxicity, tinnitus and neuropathophysiology. Acta Otorhinolaryngologica Italica. 2014;34:79-82.

17. Yankaskas K. Prelude: Noise-induced tinnitus and hearing loss in the military. Hearing Res. 2013;295:3-8.

18. Wells TS, Seelig AD, Ryan MA, Jones JM, Hooper TI, Jacobson IG, et al. Hearing loss associated with US military combat deployment. Noise \& health. 2015;17:34-6.

19. Masterson EA, Sweeney, MH, Deddens JA, Themann CL, Wall DK. Prevalence of workers with shifts in hearing by industry: A comparison of OSHA and NIOSH Hearing Shift Criteria. J Occup Environ Med. 2014;56:446-55.

20. Tantranont K, Codchanak N. Predictors of hearing protection use among industrial workers. Workplace health \& safety. 2017;65:365-71.

21. Moallemi M. Assessment of hearing state in heavy and semiheavy vehicle drivers. J Rehab Med. 2014;2:31-6.

22. Van der Molen HF, de Vries SC, Stocks SJ, Warning J, Frings-Dresen $\mathrm{MH}$. Incidence rates of occupational diseases in the Dutch construction sector, 2010-2014. Occup Environ Med. 2016;73:350-2.
23. Petrić D. Connection between noisy environment at work and the occurrence of tinnitus. 2017;1:1-6.

24. Nelson DI, Nelson RY, Concha-Barrientos M, Fingerhut M. The global burden of occupational noise-induced hearing loss. Am J Ind. 2005;48:446-58.

25. Schaafsma F, Benke G, Radi S, Sim M. Noise Induced Hearing Loss and Tinnitus: Monash University Centre for Occupational and Environmental Health. 2010.

26. Kim HJ, Lee HJ, An SY, Sim S, Park B, Kim SW, et al. Analysis of the prevalence and associated risk factors of tinnitus in adults. Plos One. 2015;10:127-578.

27. Moon IS, Park SY, Park HJ, Yang HS, Hong SJ, Lee WS. Clinical characteristics of acoustic trauma caused by gunshot noise in mass rifle drills without ear protection. $J$ Occup Environ Hyg. 2011;8:618-23.

28. Sereda M, Hall DA, Bosnyak DJ, Edmondson-Jones M, Roberts LE, Adjamian P,et al. Re-examining the relationship between audiometric profile and tinnitus pitch. Int $\mathrm{J}$ Audiol. 2011;50:303-12.

29. Martines F, Bentivegna D, Di Piazza F, Martines E, Sciacca $V$, Martinciglio $G$. Investigation of tinnitus patients in Italy: clinical and audiological characteristics. Int $\mathrm{J}$ Otolaryngol. 2010;4:1-8.

30. Faramarzi I, Kaviani M, Sadeghi Ha. Brief Communication: Prevalence Of Noise Induced Hearing Loss In Employees In Shiraz Industrial Factories. Med J Islamic Rep Iran. 2006;20:49-51. 